\title{
Punishing greediness in Divide-the-dollar games
}

\author{
Shiran Rachmilevitch*
}

July 21,2016

\begin{abstract}
Brams and Taylor (1994) presented a version of the Divide-the-dollar game (DD), which they call DD1. DD1 suffers from the following drawback: when each player demands approximately the entire dollar, then if the least greedy player is unique, then this player obtains approximately the entire dollar even if he is only slightly less greedy than the other players. I introduce a parametrized family of 2-person DD games whose "endpoints" (the games that correspond to the extreme points of the parameter space) are (1) a variant of DD1, and (2) a game that completely overcomes the greediness-related problem. I also study an $n$-person generalization of this family. Finally, I show that the modeling choice between discrete and continuous bids may have far-reaching implications in DD games.
\end{abstract}

Keywords: Bargaining games; Divide-the-dollar; Fair division.

*Department of Economics, University of Haifa, Mount Carmel, Haifa, 31905, Israel. Tel: +9724-8249588; fax: +972-4-8240059. E-mail address: shiranrach@econ.haifa.ac.il 


\section{Introduction}

Dividing a limited resource among a group of agents is one of the most basic economic problems there are. The Divide-the-dollar game, henceforth DD, is one of the simplest models of this problem. The rules of DD are as follows: each of $n$ players submits a demand (or bid) $x_{i} \in[0,1]$; if $\sum_{i=1}^{n} x_{i} \leq 1$, then each player obtains what he asked for; otherwise - i.e., if the sum of demands exceeds the available resourceno one gets anything. Each player's utility equals the amount of money he receives. ${ }^{1}$

DD has two major drawbacks: first, any split of the dollar is supported as a Nash equilibrium outcome; second, even the slightest degree of infeasibility - when the sum of demands equals $1+\epsilon$, with $\epsilon>0$ being arbitrarily small-leads to a complete waste of the social resource. These shortcomings led researchers to consider modifications of $\mathrm{DD}$ in order to overcome them.

In particular, Brams and Taylor (1994, henceforth BT) considered several variants of DD, one of which - a game they call DD1 - will be of special interest in the present paper. The rules of DD1 are as follows. Given the vector of demands, the players are partitioned into equivalence classes, according to the amount they demand. Each player in the set of the lowest bidders - the first equivalence class - receives what he asks for if there is enough money to do so; otherwise, the dollar is divided evenly among these players. If there is money left after the just-mentioned step has been applied, the second equivalence class is considered, to which the same rule is applied. This process continuous in the same fashion until (i) the dollar is exhausted, or (ii) each player received his demand. DD1 overcomes the drawbacks that were mentioned in the previous paragraph: the entire dollar is distributed to the players if the sum of

\footnotetext{
${ }^{1}$ The 2-person version of this game is a simple version of a slightly more general 2-person game, called the Nash demand game (Nash, 1953). DD also has "dual" mechanisms, where each player reports how much he thinks others should obtain; such mechanisms have been studied by De Clippel et al. (2008) for $n \geq 3$ players. A multi-stage DD game has been studied by Cetemen and Karagözoğlu (2014).
} 
demands is at least one, and the equilibrium-multiplicity problem is significantly mitigated. ${ }^{2}$ Unfortunately, DD1 suffers from a new drawback: when each player demands approximately the entire dollar, then if the least greedy player is unique, then this player obtains approximately the entire dollar even if he is only slightly less greedy than the other players. For instance, if there are two players and the demands are 0.98 and 0.99 the payoff are $(0.98,0.02)$. The main contribution of the present paper is a parametrized family of 2-person DD games that generalizes DD1. The parameter value which is associated with each family member, $\lambda \in[0,1]$, captures the degree to which the greediness-related problem can occur. The higher is $\lambda$, the smaller is the scope of the problem. When $\lambda=1$, the problem is completely solved. On the other hand, small values of $\lambda$ allow for severe punishment of the most greedy player. ${ }^{3}$

Another issue I address is the fact that the analysis in BT is carried out for the case of discrete bids. Namely, there is a minimal money-unit - a "cent" — and all demands must be multiples of it. There are two reasons to relax this assumption.

First, the "cent" takes the form $\delta=\frac{1}{n K}$, where $n$ is the number of players and $K$ is some positive integer. Therefore, the model contains an unbreakable link between the number of players and the grid of currency - two conceptually unrelated quantities.

Second, one would like to set up the environment in a way that gives some flexibility in altering the rules of the game; namely, an environment in which a variety of DD games can be defined. The discreteness assumption hurts this flexibility. To illustrate the point with a concrete example, consider the following DD game, DD*, due to Anbarci (2001). In its 2-person version, when the demands are infeasible, namely when $x_{1}+x_{2}>1$, each player $i$ receives the payoff $f_{i} x_{i}$, where $f_{i} \equiv \frac{1-x_{i}}{x_{j}}$. The

\footnotetext{
${ }^{2}$ DD1 has multiple Nash equilibria, but they are all payoff-equivalent. In any equilibrium each player obtains the egalitarian utility level.

${ }^{3}$ Abusing terminology a little, I call any divide-the-dollar game a DD game. A general DD game is a game which is identical to the canonical DD that was described in the first paragraph of this Introduction, with the sole exception that when the sum of demands exceeds one, the dollar need not be wasted - it (or a fraction of it) can be distributed in some way among the players.
} 
economic rationale behind this rule is as follows. When player 1 makes his demand, he offers player 2 the amount $1-x_{1}$. Since player 2 demands $x_{2}$, it is as if player 1 asks player 2 to settle for a fraction $f_{1}$ of what player 2 asks for himself, where $f_{1} \equiv \frac{1-x_{1}}{x_{2}}$. Similarly, player 2 asks player 1 , effectively, to settle for a fraction $f_{2}$ of what player 1 asks for himself, where $f_{2} \equiv \frac{1-x_{2}}{x_{1}}$. Now, if it is legit to expect your fellow bargainer to settle for a fraction $f$ of his demand, then, one may argue, it is perfectly fine to require that you, the proposer, would also settle for this fraction out of what you wish for yourself. This is the logic of Anbarci's game. Since the $f_{i}$ 's are, by definition, fractions, $\mathrm{DD}^{*}$ is not well-defined under discrete demands. For example, consider the most basic case, where the minimal money-unit is 0.01 ; here, if player 1 demands $x_{1}=0.6$ and player 2 demands $x_{2}=0.51$, then player 1 's payoff is $f_{1} x_{1}=\frac{0.24}{0.51}$, which is not a multiple of cents.

In reality money is discrete, hence one may argue that this discreteness should be reflected in the model. My point is that even though discreteness is realistic, it expresses a constraint which should not be at the center stage of the analysis. The money-continuity assumption, which is typical to bidding games, is therefore in place.

The rest of the paper is organized as follows. In Section 2 I present a list of conditions for DD games, due to BT, called reasonableness conditions. In Section 3 I revisit DD1, but, as opposed to BT, under the assumption that money is continuous (an assumption I will maintain throughout the paper). I denote the DD1 game under continuous demands by cDD1. Both DD1 and cDD1 are reasonable. The choice between continuity and discreteness of money has far-reaching implications. In DD1 there are multiple (payoff equivalent) Nash equilibria and an equilibrium can be arrived by iteratively removing weakly dominated strategies; additionally, in the 2-person DD1, as in any 2-person reasonable DD game with discrete demands, the egalitarian demand level is weakly dominated. ${ }^{4}$ By contrast, in cDD1 the vector of egalitarian demands is the unique Nash equilibrium, and the egalitarian demand level

\footnotetext{
${ }^{4}$ See Theorem 1 in BT.
} 
is not a weakly dominated strategy. In Section 4 I present the family of 2-person DD

games $\left\{\mathrm{DD}^{\lambda}\right\}_{\lambda \in[0,1]}$. The generalization of $\left\{\mathrm{DD}^{\lambda}\right\}_{\lambda \in[0,1]}$ to $n$ bidders is in Section 5 . In Section 6 I conclude.

\section{The reasonableness conditions}

BT specify five conditions for DD games, which are satisfied, in particular, by DD1. They call any DD game that respects these conditions reasonable. The conditions are:

(1) Equal treatment of equal demands.

(2) No player's payoff is more than his demand.

(3) If the sum of the demands does not exceed one, then each player receives his demand.

(4) If the sum of the demands exceeds one, then the dollar, nevertheless, is completely disbursed to the players.

(5) If all demands are greater than the egalitarian level demand, the player with the highest demand does not receive a higher payoff than the player with the lowest demand.

\section{3 cDD1: the DD1-game with continuous demands}

Suppose that each player can submit any demand in $[0,1]$. In particular, there is no minimal money-unit. I now define a DD1-type game for this environment. To this end, the following notation and definitions will be helpful.

Every profile of demands, $x=\left(x_{1}, \cdots, x_{n}\right) \in[0,1]^{n}$, can be partitioned into equivalence classes according to the values of its coordinates. An equivalence class consists of the coordinates that assume the same value; namely, it is a set of the form $\left\{i, j: x_{i}=x_{j}\right\}$. Suppose that these classes are labeled "from the bottom up," 
so higher demands belong to higher-indexed classes. Let $k_{i}$ and $I_{i}$ be the following functions of demand: $k_{i}(x)$ denotes the number of class to which player/coordinate $i$ belongs (so $x_{i}<x_{j}$ implies $k_{i}(x)<k_{j}(x)$ ) and $I_{i}(x)$ denotes the number of players/coordinates that are in the same equivalence class as player/coordinate $i$. The continuous version of DD1 - hereafter cDD1 - is the DD game the utility functions of which are the following:

$u_{i}^{c D D 1}(x) \equiv \begin{cases}x_{i} & \text { if } \sum_{\left\{j: k_{j}(x) \leq k_{i}(x)\right\}} x_{j} \leq 1 \\ \frac{1}{I_{i}(x)} & \text { if } k_{i}(x)=1 \text { and } \sum_{\left\{j: k_{j}(x)=1\right\}} x_{j}>1 \\ \frac{1-\sum_{\left\{j: k_{j}(x)<k_{i}(x)\right\}} x_{j}}{I_{i}(x)} & \text { if } k_{i}(x)>1 \text { and } \sum_{\left\{j: k_{j}(x)<k_{i}(x)\right\}} x_{j}<1 \leq \sum_{\left\{j: k_{j}(x) \leq k_{i}(x)\right\}} x_{j} \\ 0 & \text { otherwise }\end{cases}$

Proposition 1. cDD1 is a reasonable DD game. The profile $\left(\frac{1}{n}, \cdots, \frac{1}{n}\right)$ is its unique Nash equilibrium. The egalitarian demand, $x_{i}=\frac{1}{n}$, is not a weakly dominated strategy in this game.

Proof. Note that conditions (1) and (3)-(5) are straightforward. As for condition (2), note that the only way for player $i$ to obtain a payoff above $x_{i}$ is if his payoff obeys the third line from the definition of the utility function. In this case, to have the payoff exceed the demand one needs $\frac{1-\sum_{\left\{j: k_{j}(x)<k_{i}(x)\right\}} x_{j}}{I_{i}(x)}>x_{i}$, or $1-\sum_{\left\{j: k_{j}(x)<k_{i}(x)\right\}} x_{j}>$ $x_{i} I_{i}(x)$, which contradicts $1 \leq \sum_{\left\{j: k_{j}(x) \leq k_{i}(x)\right\}} x_{j}$. Therefore, cDD1 is reasonable.

As for equilibrium uniqueness, first note that $\left(\frac{1}{n}, \cdots, \frac{1}{n}\right)$ is indeed an equilibrium: any demand deviation downward decreases one's payoff and any deviation up leaves it unchanged. Now let $x$ be an arbitrary equilibrium. Let $l$ be the common demand in its first equivalence class (namely, $l$ is the minimum demand in $x$ ).

Case 1: $x_{i}=l$ for all $i$. It has to be that $\ln \geq 1$; namely, the demands exhaust the dollar. Otherwise, if $\ln <1$, then each player could increase his payoff by deviating to $l+\Delta$, where $\Delta=1-(n-2) l($ so $l+\Delta=1-(n-1) l)$. Therefore $l n \geq 1$, or $l \geq \frac{1}{n}$. Next, I argue that $l>\frac{1}{n}$ is impossible. The reason is that in this case each player could increase his payoff by deviating to some $l^{\prime} \in\left(\frac{1}{n}, l\right)$. Therefore, $x=\left(\frac{1}{n}, \cdots, \frac{1}{n}\right)$. 
Case 2: There is an $i$ with $x_{i}>l$. Let $m$ be the common bid of the second equivalence class. Let $k$ be the number of players in the first equivalence class. I argue that $k l \geq 1$; namely, the sum of minimal demands exhausts the dollar. Otherwise, each of the lowest bidders could strictly benefit by deviating to $l+\Delta$, for $\Delta \in(0, m-l)$. Note that by doing that a deviator becomes a singleton second equivalence class and his payoff, consequently is one of the following two: (a) $l+\Delta>l$-in case that his demand can be met after all the $k-1$ minimal bidders have been paid; or (b) $1-(k-1) l>l$-in case that there is not enough money left to satisfy his demand. Thus, $k l \geq 1$. Next, I argue that $k l>1$ is impossible; this is due to the same reason as in Case 1-in such a case an infinitesimal deviating downwards is strictly profitable for each of the minimal bidders. Therefore $l=\frac{1}{k}$, and the dollar is shared equally between the minimal bidders. But this, in turn, means that each $i$ with $x_{i}>l$ is not playing a best-response. Therefore, $\left(\frac{1}{n}, \cdots, \frac{1}{n}\right)$ is the unique equilibrium.

Finally, we need to prove that $x_{i}=\frac{1}{n}$ is not weakly dominated; wlog, consider $i=1$. Assume by contradiction that the egalitarian demand is dominated for player 1. Let $z$ be some other demand that weakly dominates the egalitarian demand. Since it is possible for all other players to demand zero, it has to be that $z>\frac{1}{n}$. Pick a $y \in\left(\frac{1}{n}, \min \left\{z, \frac{1}{n-1}\right\}\right)$. Weak domination implies:

$$
u_{1}(z, y, y, \cdots, y) \geq u_{1}\left(\frac{1}{n}, y, y, \cdots, y\right)
$$

By the rules of the game, the LHS equals $1-(n-1) y$ and the RHS equals $\frac{1}{n}$. Hence, we obtain the contradiction $y \leq \frac{1}{n}$.

Proposition 1 shows that there is a significant difference between the current model and the discrete-money model. In the latter, any reasonable 2-person DD game is such that the egalitarian demand level is a weakly dominated strategy. In particular, this applies to DD1. By contrast, the egalitarian demand level is not weakly dominated in cDD1. Moreover, DD1 has multiple Nash equilibria, whereas cDD1 has a unique equilibrium. 


\section{Punishing greediness: A generalization of the 2-person cDD1}

When there are two players, both versions of the game-DD1 and cDD1-suffer from the following drawback. According to the rules of these games, when both players are extremely greedy, a tiny difference in their demands makes the player who is slightly less greedy receive an overwhelming share of the dollar. For instance, if the demands are 0.98 and 0.99 the payoff are $(0.98,0.02)$.

Say that a 2-person DD game with utility functions $\left\{u_{1}, u_{2}\right\}$ has an inequality parameter $\gamma$ if:

$$
\gamma \equiv \lim _{\epsilon \downarrow 0}\left[u_{2}(1-\epsilon, 1-2 \epsilon)-u_{1}(1-\epsilon, 1-2 \epsilon)\right]
$$

A small value of $\gamma$ prevents the aforementioned problem, as a small $\gamma$ means that when the two players are extremely greedy (each demands, approximately, the entire dollar) then there may be discrimination against the one who is more greedy, but there are limits to discrimination; specifically, the limits of discrimination are given by $\gamma$. Note that $\gamma$, by definition, is between zero and one. Therefore, cDD1's performance in this regard is the worst possible, since its inequality parameter is $\gamma=1 .^{5}$

While a low value of $\gamma$ is obviously desirable, a high value of $\gamma$ also has an upside, at least under condition (4) from the BT list. Note that when the demands are jointly infeasible and one player is more greedy than the other, we may wish to punish this player, which means giving him a low payoff; but by condition (4) this means giving a relatively high payoff to the other player, which, in turn, results in a high value for the inequality parameter. Therefore, there is a tradeoff between two competing principles: (i) similar treatment of greedy players, versus (ii) awarding the least greedy player. ${ }^{6}$

\footnotetext{
${ }^{5}$ For cDD1 we have $u_{2}(1-\epsilon, 1-2 \epsilon)-u_{1}(1-\epsilon, 1-2 \epsilon)=1-2 \epsilon-2 \epsilon$. Therefore $\gamma=1$ for this game.

${ }^{6}$ In the 2-person case principle (ii) is equivalent to punishing the most greedy player.
} 
Below is a parametrized family of 2-person DD games, in which the parameter that corresponds to each family member captures this tradeoff.

Given $\lambda \in[0,1]$, let $\mathrm{DD}^{\lambda}$ denote the 2-person DD game in which the utility of player 1 is as follows and that of player 2 is defined analogously:

$$
u_{1}^{\lambda}(x, y) \equiv \begin{cases}x & \text { if } x+y \leq 1 \quad \text { (i) } \\ \frac{\lambda x}{1+x} & \text { if } x+y>1 \text { and } x>y \geq \frac{1+(1-\lambda) x}{1+x} \\ \frac{1+(1-\lambda) y}{1+y} & \text { if } x+y>1 \text { and } y>x \geq \frac{1+(1-\lambda) y}{1+y} \\ \frac{1}{2} & \text { if } x+y>1 \text { and } x=y \text { (iv) } \\ u_{1}^{c D D 1}(x, y) & \text { otherwise (v) }\end{cases}
$$

In words, the rules of the game are as follows. If the demands are jointly feasible then, like in any DD game, each player receives his demand. If not, then the players split the dollar evenly in case they submitted equal demands; if they submitted different demands then the player who is more greedy receives $\frac{\lambda z}{1+z}$ where $z$ is his demand, but only if giving the rest of the dollar to the other player does not make the other player end up with an amount that exceeds his demand; in any other case the rules of cDD1 are implemented. It is easy to verify that $\mathrm{DD}^{\lambda}$ is reasonable for every $\lambda \in[0,1]$. Also, note that $\mathrm{DD}^{0}=\mathrm{cDD} 1$, because with $\lambda=0$ (ii) and (iii) can never be realized. Therefore, $\left\{\mathrm{DD}^{\lambda}\right\}_{\lambda \in[0,1]}$ is a generalization of $\mathrm{cDD} 1$.

Proposition 2. For each $\lambda \in[0,1)$, the game $D D^{\lambda}$ has a unique Nash equilibrium: $\left(\frac{1}{2}, \frac{1}{2}\right)$.

Proof. The case $\lambda=0$ was covered in Theorem 1. Fix then a $\lambda \in(0,1)$ and consider the corresponding game. First, let us verify that $\left(\frac{1}{2}, \frac{1}{2}\right)$ is indeed an equilibrium. Wlog, consider player 1. Obviously, decreasing his demand is suboptimal. If he increases it then the resulting case is either (ii) or (v). In the former case his payoff is $\frac{\lambda x}{1+x} \leq \frac{\lambda}{2}<\frac{1}{2}$. In the latter case his payoff is without a change $-\frac{1}{2}$. Therefore, $\left(\frac{1}{2}, \frac{1}{2}\right)$ is a Nash equilibrium.

Now let $(x, y)$ be an equilibrium. Clearly, $x+y \geq 1$. I argue that $x+y=1$. To 
see this, assume by contradiction that $x+y>1$. Suppose first that $x \neq y$; wlog, that $x>y$. Either (ii) or (v) hold. If (ii) is the case then $x=1$ and therefore player 1's payoff is $\frac{\lambda}{2}$, which is smaller than $\frac{1}{2}$ - which is what player 1 can achieve by playing $y$. If $(\mathrm{v})$ is the case then $y<\frac{1+(1-\lambda) x}{1+x}$, which is impossible in equilibrium, because player 2 could increase his demand by a sufficiently small $\epsilon>0$ and increase his payoff (note that as long as we are in case (v) player 2's payoff equals his demand, since it is given by the payoff rule of cDD1). Therefore, if $x+y>1$ then $x=y$. This, again, cannot occur in equilibrium because by a small decrease of his demand player 1 can increase his payoff. To see this, let us consider two cases separately.

Case 1: If $y>\frac{1+(1-\lambda) y}{1+y}$ then by an infinitesimal decrease of his demand player 1 can obtain a payoff which is approximately $\frac{1+(1-\lambda) y}{1+y}$, and $\frac{1+(1-\lambda) y}{1+y}>\frac{1}{2} \cdot{ }^{7}$

Case 2: $y \leq \frac{1+(1-\lambda) y}{1+y}$. Here, by deviating to $y-\epsilon$ player 1 triggers the DD1 payment rule, and therefore obtains $y-\epsilon>\frac{1}{2}$.

Therefore, if $(x, y)$ is an equilibrium, then $x+y=1$.

Now suppose, wlog, that $x<\frac{1}{2}$. Then player 1 has a profitable deviation: from $x$ to $1-x$. Therefore, $\left(\frac{1}{2}, \frac{1}{2}\right)$ is the unique equilibrium.

As $\lambda$ increases the most greedy player is being penalized less severely. The inequality parameter of $\mathrm{DD}^{\lambda}$ is $\gamma=1-\lambda$. Hence, there is a tradeoff between minimizing the inequality parameter and decreasing the incentive to be greedy. This tradeoff results in the fact that when the inequality parameter is minimized, extreme greediness can occur in equilibrium.

Proposition 3. The set of $D D^{1}$ 's Nash equilibria is $\left\{\frac{1}{2}, 1\right\} \times\left\{\frac{1}{2}, 1\right\}$.

Proof. That $\left(\frac{1}{2}, \frac{1}{2}\right)$ is an equilibrium follows from precisely the same argument as in the proof of Proposition 1.

Consider $(1,1)$. By the rules of the game (the demands are in the unit interval),

\footnotetext{
${ }^{7}$ This inequality is equivalent to $2+2(1-\lambda) y>1+y$ or $1+y[2(1-\lambda)-1]>0$. The latter inequality is true because its LHS, which is strictly decreasing in $\lambda$ is strictly greater than $1-y \geq 0$.
} 
the only possible deviation is downwards. Consider, wlog, such a deviation by player 1. The possible cases that player 1 can trigger by his deviation are (i), (iii), and (v). Obviously triggering (i) - namely, deviation to 0 - is suboptimal. In case (iii) player 1 's payoff does not change - it remains $\frac{1}{2}$. Finally, in order to trigger (v) it needs to be that the demand to which player 1 deviates, call it $x$, satisfies $x<\frac{1}{2}$; since in this case player 1's payoff receives his demanded amount (by the rules of cDD1), the deviation is strictly suboptimal. Therefore, $(1,1)$ is an equilibrium.

Now consider $\left(1, \frac{1}{2}\right)$. A deviation by player 1 that causes (i) is clearly unprofitable, and conditional on (ii) $x=1$ is optimal. Case (iii) is impossible since $y=\frac{1}{2}$, and in case (iv) player 1's payoff remains $\frac{1}{2}$. Finally, (v) is also sub-optimal: since player 2 demands $y=\frac{1}{2}$, player 1 cannot get more than $\frac{1}{2}$ under the cDD1 rules. Therefore player 1 does not have profitable deviation. Now consider player 2. A deviation that triggers (i) is a deviation to zero and is clearly sub-optimal. Conditional on (ii), player 2's payoff is without a change. Case (iii) is impossible since $x=1$. A deviation to $y=1$-namely to case (iv) — does not change his payoff. Finally, under (v) player 2 obtains his demand, but since $x=1$ it follows that (v) can be realized only if player 2's demand is below $\frac{1}{2}$. Therefore $\left(1, \frac{1}{2}\right)$ is an equilibrium. Similarly, $\left(\frac{1}{2}, 1\right)$ is an equilibrium.

Now let $(x, y)$ be an arbitrary equilibrium. Consider first $x \neq y$; wlog, $x>y$. Clearly, (i) is impossible. Therefore, $x+y>1$. Also, (v) is impossible, since in this case player 2 can increase his payoff by increasing his demand. Cases (iii) and (iv) are impossible and we are therefore led to (ii), and therefore to $x=1$. If $y \in\left\{\frac{1}{2}, 1\right\}$, we are in one of the equilibria encountered above. If not, then $y \in\left(\frac{1}{2}, 1\right)$. If $y \leq \frac{-1+\sqrt{5}}{2}$, then by deviating to $x^{\prime} \in\left(\frac{1}{2}, y\right)$ player 1 triggers case (v) and increases his payoff to $x^{\prime}>\frac{1}{2}$. If $y>\frac{-1+\sqrt{5}}{2}$ then by deviating to $x^{\prime \prime} \in\left(\frac{1}{1+y}, y\right)$ player 1 triggers case (iii) and increases his payoff to $\frac{1}{1+y}>\frac{1}{2}$. Finally, consider the case $x=y \equiv z$. Clearly $z \geq \frac{1}{2}$. If $z \in\left\{\frac{1}{2}, 1\right\}$, we are in one of the equilibria encountered above. If not, then $z \in\left(\frac{1}{2}, 1\right)$. If $z \leq \frac{-1+\sqrt{5}}{2}$, then by deviating to $x^{\prime} \in\left(\frac{1}{2}, z\right)$ player 1 triggers case (v) 
and increases his payoff to $x^{\prime}>\frac{1}{2}$. If $z>\frac{-1+\sqrt{5}}{2}$ then by deviating to $x^{\prime \prime} \in\left(\frac{1}{1+z}, z\right)$ player 1 triggers case (iii) and increases his payoff to $\frac{1}{1+z}>\frac{1}{2}$.

\section{An $n$-person generalization of $\mathrm{DD}^{\lambda}$}

Here is a generalization of $\left\{\mathrm{DD}^{\lambda}\right\}_{\lambda \in[0,1]}$ to an arbitrary numbers of players, $n \geq 2$. Let $\Pi$ be the set of permutations on $\{1, \cdots, n\}$. Fix $\theta \in\left[0, \frac{1}{n-1}\right]$. For each $\pi \in \Pi$ let $X_{\theta}^{\pi} \subset[0,1]^{n}$ be the set of demand vectors $x$ that satisfy the following four conditions:

- (I) $\sum_{i} x_{i}>1$

- (II) $x_{\pi(1)} \geq x_{\pi(2)} \geq \cdots \geq x_{\pi(n-1)}>x_{\pi(n)}$.

- (III) $x_{\pi(k)} \geq \frac{\theta x_{\pi(1)}}{1+x_{\pi(1)}}$ for all $k=1, \cdots, n-1$.

- (IV) $x_{\pi(n)} \geq \frac{1+[1-(n-1) \theta] x_{\pi(1)}}{1+x_{\pi(1)}}$.

Define $D D^{\theta}$ to be the $n$-person DD game with the utility functions $\left\{u_{1}^{\theta}, \cdots, u_{n}^{\theta}\right\}$ that are defined as follows. On $[0,1]^{n} \backslash\left(\cup_{\pi \in \Pi} X_{\theta}^{\pi}\right), u_{i}^{\theta}=u_{i}^{c D D 1}$. On $X_{\theta}^{\pi}, u_{i}^{\theta}$ is defined by:

$$
u_{i}^{\theta}(x) \equiv \begin{cases}\frac{\theta x_{\pi(1)}}{1+x_{\pi(1)}} & \text { if } i \neq \pi(n) \\ \frac{1+[1-(n-1) \theta] x_{\pi(1)}}{1+x_{\pi(1)}} & \text { otherwise }\end{cases}
$$

To make this definition more transparent, let us look at the case where $\pi$ is the identity. Consider then $x \in X_{\theta}^{\pi}$ where $\pi$ is the identity. Each $i<n$ obtains $\frac{\theta x_{1}}{1+x_{1}}$ and player $n$ obtains the remainder of the dollar, $\frac{1+[1-(n-1) \theta] x_{1}}{1+x_{1}}$. Note that for $n=2$ $\mathrm{DD}^{\theta}=\mathrm{DD}^{\lambda}$, where $\lambda=\theta$. Analogously to $\lambda=0$ from the 2-person case, $\theta=0$ corresponds to cDD1.

It is easy to verify that $\mathrm{DD}^{\theta}$ is reasonable and that $\left(\frac{1}{n}, \cdots, \frac{1}{n}\right)$ is an equilibrium of this game. The following result describes an additional condition under which it is the game's unique equilibrium.

Proposition 4. Let $\theta \in\left[0, \frac{2}{2 n-1}\right)$. Then $\left(\frac{1}{n}, \cdots, \frac{1}{n}\right)$ is $D D^{\theta}$ 's unique Nash equilibrium. 
Proof. Let $\theta$ be as above. Let $\theta_{n} \equiv 1-(n-1) \theta$. By assumption, $\theta_{n}>\frac{1}{2 n-1}$. For each $\pi \in \Pi$ let $D_{\theta}^{\pi}$ be the set of demand vectors that are one deviation away from $X_{\theta}^{\pi}$. That is, $x \in D_{\theta}^{\pi}$ if and only if $x \notin X_{\theta}^{\pi}$ and there exist a player $i$ and a vector $x^{\prime} \in X_{\theta}^{\pi}$ such that $x_{j}=x_{j}^{\prime}$ for all $j \neq i$.

Let $x$ be an equilibrium of $D D^{\theta}$. I argue that there does not exist a $\pi \in \Pi$ such that $x \in X_{\theta}^{\pi} \cup D_{\theta}^{\pi}$. Assume by contradiction that such a $\pi$ exists. Wlog, suppose that it is the identity.

Case 1: $x \in X_{\theta}^{\pi}$. Since $x$ is an equilibrium, $x_{1}=1$. The payoff for player $i<n$ is $\frac{\theta}{2}$. I argue that every such $i$ has a profitable deviation. Note that when he deviates and ties with the minimum bidder (i.e., the deviation is $x_{i} \mapsto x_{n}$ ) he secures the payoff $\min \left\{\frac{1}{2}, x_{n}\right\}$, because he triggers the cDD1 payment rule. Thus, if $x_{n} \geq \frac{1}{2}$ he secures the payoff $\frac{1}{2}$; the deviation is profitable, since $\frac{1}{2}>\frac{\theta}{2}$. Suppose then that $\frac{1}{2}>x_{n}$. Since every $i$ should find the abovementioned deviation non-profitable, it follows that $x_{n} \leq \frac{\theta}{2}$. Therefore $(n-1) x_{n} \leq \frac{1}{2}\left(1-\theta_{n}\right)$. Since $x \in X_{\theta}^{\pi}$ and $x_{1}=1$ it follows that $x_{n} \geq \theta_{n}{ }^{8}$ Therefore $(n-1) \theta_{n} \leq \frac{1}{2}\left(1-\theta_{n}\right)$. Rearranging this gives $\theta_{n} \leq \frac{1}{2 n-1}$, a contradiction.

Case 2: $x \in D_{\theta}^{\pi}$. First, note that the minimum demand is not unique. To see this, assume by contradiction that there is a permutation $\eta$ such that $x_{\eta(1)} \geq x_{\eta(2)} \geq$ $\cdots \geq x_{\eta(n-1)}>x_{\eta(n)}$. Wlog, suppose that $\eta$ is the identity.

Claim 1: $x_{n}<\frac{1+\theta_{n} x_{1}}{1+x_{1}}$.

Proof of Claim 1: Assume by contradiction that $x_{n} \geq \frac{1+\theta_{n} x_{1}}{1+x_{1}}$. Since $x$ is an equilibrium, $\sum x_{i} \geq 1$. Moreover, this inequality is strict. If not then $x_{n}<\frac{1}{n}$, and we obtain:

$$
\frac{1+\theta_{n}}{2} \leq \frac{1+\theta_{n} x_{1}}{1+x_{1}} \leq x_{n}<\frac{1}{n},
$$

hence $\theta_{n}<\frac{2}{n}-1 \leq 0$, a contradiction. Therefore, condition (I) holds. Since (II) holds by assumption and (IV) holds by assumption, it follows that (III) is violated. Namely,

$$
{ }^{8} x_{n} \geq \frac{1+\theta_{n}}{2} \text { and } \frac{1+\theta_{n}}{2} \geq \theta_{n} .
$$


there is an $i<n$ such that $x_{i}<\frac{\theta x_{1}}{1+x_{1}}$. Since $x_{i}>x_{n}$ we obtain the contradiction $\frac{\theta x_{1}}{1+x_{1}}>x_{i}>x_{n} \geq \frac{1+\theta_{n} x_{1}}{1+x_{1}}$. Claim 1 is proved.

It follows from Claim 1 that if player $n$ increases his demand by a sufficiently small amount then the resulting demand vector would still be outside of $\cup_{\theta} X_{\theta}$ and hence the payment rule would still be as in cDD1. Therefore, this deviation is profitable. Thus, there is a "tie" at the minimum demand. Moreover, only two players are tied at the minimum demand level; otherwise, the demand vector would not be one deviation away from $\cup_{\theta} X_{\theta}$. Therefore, $x_{1} \geq \cdots \geq x_{n-2}>x_{n-1}=x_{n}$. Let $t \equiv x_{n}=x_{n-1}$.

\section{Claim 2: $t \geq \frac{1+\theta_{n} x_{1}}{1+x_{1}}$.}

Proof of Claim 2: Recall that $\cup_{\theta} X_{\theta}$ is reachable by one deviation. Consider the set of all such deviations. If there does not exist such a deviation in which the deviator is player 1 and he undercuts $t$, then necessarily $t \geq \frac{1+\theta_{n} x_{1}}{1+x_{1}}$. The reason is that after such a deviation the maximal bid remains $x_{1}$ and the minimum bid strictly decreases. The new (post deviation) minimum has to be at least as large as $\frac{1+\theta_{n} x_{1}}{1+x_{1}}$. Suppose, on the other hand, that there is a deviation in which player 1 is the deviator. In this case, the new (post deviation) maximum is $x_{2}$. Call the new minimum $t^{\prime}$. Clearly $t^{\prime}<t$. Therefore, $t<\frac{1+\theta_{n} x_{1}}{1+x_{1}}$ implies $t^{\prime}<\frac{1+\theta_{n} x_{1}}{1+x_{1}}$. Since the new demand vector must respect conditions (I)-(IV), it follows that $\frac{1+\theta_{n} x_{2}}{1+x_{2}} \leq t^{\prime}$ and therefore $\frac{1+\theta_{n} x_{2}}{1+x_{2}}<\frac{1+\theta_{n} x_{1}}{1+x_{1}}$ implying that $x_{2}>x_{1}-$ a contradiction. Claim 2 is proved.

Now, since $t \geq \frac{1+\theta_{n} x_{1}}{1+x_{1}} \geq \frac{1+\theta_{n}}{2}>\frac{1}{2}$, the dollar is divided evenly between players $\{n, n-1\}$. By deviating to $t-\epsilon$, for a sufficiently small $\epsilon>0$, player $i \in\{n, n-1\}$ secures a payoff of at least $\min \left\{t-\epsilon, \frac{1+\theta_{n} x_{1}}{1+x_{1}}\right\}>\frac{1}{2}$.

Therefore, as argued, there does not exist a $\pi \in \Pi$ such that $x \in X_{\theta}^{\pi} \cup D_{\theta}^{\pi}$.

Now assume by contradiction that the equilibrium, $x$, satisfies $x \neq\left(\frac{1}{n}, \cdots, \frac{1}{n}\right)$. By Proposition 1, $x$ is not an equilibrium of cDD1. Therefore, under the cDD1 rule there is a player, say $h$, who has a profitable deviation. Let $x^{\prime}$ be the demand vector that results from the deviation. Therefore, $u_{h}^{c D D 1}\left(x^{\prime}\right)>u_{h}^{c D D 1}(x)$. Since $x \notin \cup_{\pi \in \Pi} X_{\theta}^{\pi}$ it follows that $u_{h}^{\theta}(x)=u_{h}^{c D D 1}(x)$. Since $x \notin \cup_{\pi \in \Pi} D_{\theta}^{\pi}$ it follows that 
$x^{\prime} \notin \cup_{\pi \in \Pi} X_{\theta}^{\pi}$. Therefore $u_{h}^{\theta}\left(x^{\prime}\right)=u_{h}^{c D D 1}\left(x^{\prime}\right)$. Therefore $u_{h}^{c D D 1}\left(x^{\prime}\right)>u_{h}^{c D D 1}(x)$ implies $u_{h}^{\theta}\left(x^{\prime}\right)>u_{h}^{\theta}(x)$; hence $x$ is not an equilibrium of $\mathrm{DD}^{\theta}$ - a contradiction.

The inequality parameter for the $n$-person case is defined analogously to the 2 -person case:

$$
\gamma \equiv \lim _{\epsilon \downarrow 0}\left[u_{n}(1-\epsilon, 1-2 \epsilon, \cdots, 1-n \epsilon)-u_{1}(1-\epsilon, 1-2 \epsilon, \cdots, 1-n \epsilon)\right] .
$$

As we noted, $\theta=0$ is analogous to $\lambda=0$ from the 2-person case, and the corresponding inequality parameter assumes the value $\gamma=1$. For $\theta$ such that $\theta_{n}<1$ the value of $\mathrm{DD}^{\theta}$ 's inequality parameter is $\frac{1+\theta_{n}-\theta}{2}$, the minimum of which is obtained at $\theta=\frac{1}{n-1}$. Thus, the inequality parameter is bounded from below by $\frac{n-2}{2(n-1)}$. As $n \rightarrow \infty$ the set of $\mathrm{DD}^{\theta}$ 's converges to $\mathrm{cDD} 1$ and the lower bound on the inequality parameter converges to $\frac{1}{2}$. In this sense, solving the greediness problem by employing $\mathrm{DD}^{\theta}$ becomes increasingly more difficult as the number of players increases.

\section{Conclusion}

DD games involve a tradeoff between two competing principles: (i) similar treatment of greedy players, versus (ii) awarding the least greedy player. I have captured this tradeoff parametrically by the family $\left\{\mathrm{DD}^{\lambda}\right\}_{\lambda \in[0,1]}$, and by its $n$-person generalization. A secondary and more technical contribution of the paper has been in pointing out to the fact that the choice between continuous and discrete money may have substantial implications in DD games.

Acknowledgments: I would like to thank Amnon Maltz for helpful comments. 


\section{References}

[1] Anbarci, N. (2001), Divide-the-dollar game revisited, Theory and Decision, 50, 295-303.

[2] Brams, S.J. and Taylor, A.D., (1994), Divide the dollar: Three solutions and extensions, Theory and Decision, 37, 211-231.

[3] Cetemen, E. D., and Karagözoğlu, E., (2014), Implementing equal division with an ultimatum threat, Theory and Decision, 77, 223-236.

[4] De Clippel, G., Moulin, H., and Tideman, N., (2008), Impartial division of a dollar, Journal of Economic Theory, 139, 176-191.

[5] Nash, J. (1953), Two-person cooperative games, Econometrica, 21, 128-140. 